\title{
A Universal Locked Nucleic Acid-integrated X-shaped DNA Probe Design for Amplified Fluorescence Detection of Single-nucleotide Variant
}

Fang $\mathrm{Wu}^{\mathrm{a} 1}$, Mei Chen ${ }^{\mathrm{b} 1}$, Jianming Lan ${ }^{\mathrm{a}}$, Yaokun Xia ${ }^{\mathrm{b}}$, Mengmeng Liu ${ }^{\mathrm{b}}$, Wenhui He${ }^{\mathrm{b}}$, Chunyan $\mathrm{Li}^{\mathrm{a}}$, Xiaosong Chen ${ }^{\mathrm{c} *}$, Jinghua Chen ${ }^{\mathrm{b} *}$

${ }^{a}$ Department of Basic Chemistry, the School of Pharmacy, Fujian Medical University, Fuzhou, Fujian Province 350108, China.

${ }^{b}$ Department of Pharmaceutical Analysis, the School of Pharmacy, Fujian Medical University, Fuzhou, Fujian Province 350108, China.

${ }^{c}$ Department of Plastic Surgery, the Union Hospital of Fujian Medical University, Fuzhou, Fujian Province 350108, China.

${ }^{*}$ Corresponding author. E-mail addresses: cjh_huaxue@126.com (J.-H. Chen), chenxiaosong74@163.com(X. Chen).

${ }^{1}$ Authors with equal contributions. 


\section{Abstract}

Single-nucleotide variant (SNV) is crucial in clinical diagnostics as a useful biomarker, because it is closely associated with many diseases, such as genetic disorders and tumors. In this study, an enzyme-free and selective fluorescence biosensor is proposed for $\beta$-thalassemia SNV detection based on X-shaped probe, locked nucleic acid (LNA), and toehold-mediated strand-displacement reaction (TMSDR)-assisted recycling technology. On one hand, the LNA-integrated X-shaped probe has high recognition ability for SNV sequence even in the presence of a large amount of wild-type sequence, i.e., the rare SNV can be detected down to $0.01 \%$ variant allele frequency (VAF). On the other hand, the introduction of TMSDR-assisted recycling amplification system remarkably improves the sensitivity of the sensor with the detection limit as low as $6 \mathrm{fM}$ mutation gene. Moreover, the designed $\mathrm{X}$-shaped probe is universal because its functionalized oligonucleotides could be decoupled from the target-specific regions. These experimental results demonstrate that this developed method offers high specificity and sensitivity for SNV detection, which might be a potential alternative as an effective mutation detection technology for the detection of $\beta$-thalassemia SNV and other gene-related diseases. 
Keywords: Single-nucleotide variant, X-shaped DNA probe, locked nucleic acid, toehold-mediated strand-displacement reaction, $\beta$-thalassemia, fluorescence detection. 


\section{Introduction}

Detection of single-nucleotide variants $(\mathrm{SNV})$ is crucial in clinical diagnostics, because it is closely associated with many diseases, including genetic disorders and tumors [1,2]. Recently, SNV have gained prominence as useful biomarkers at a low variant allele frequency (VAF) in cancer molecular diagnostics [3-5]. SNV detection typically depends on a mechanism for allele discrimination. Up to now, many methods, such as allele-specific hybridization methods, have been developed for SNV detection, but complicated optimization and rigorous control of the assays made direct sequencing sub-optimal $[6,7]$. On the other side, allele-specific enzymatic methods, including ligase-based ligation [8-10], endonuclease-based cleavage [11], primer extension [12-14], polymerase based nucleotide incorporation [15] and proof-reading $[16,17]$, have become a prevailing option for SNV detection because of its specificity and robustness. However, the inherent shortcomings of enzymes, such as the activity sensitive to reaction conditions and high cost, limit their application [18]. To address these problems, several enzyme-free biosensors have been presented on the basis of toehold-mediated strand-displacement reaction (TMSDR) for SNV detection [19-22]. Furthermore, in order to improve the sensitivity, many TMSDR-based recycling amplification methods have been 
adopted in recent years [23-25]. However, in these assays, the sensing system is a turn-off mode to detect the single-base mismatch, which might lead to low specificity since other quenchers or environment factors cause undesired false positive signals. Besides, the elaborate design of hairpin structure is a complicated process. Consequently, it is still essential to find a novel technology to enhance the specificity of SNV detection and some new concepts for nucleic acid probe design have been reported [26-31]. Among these reports, Wang et al. [31] developed a simulation-guided DNA probe to assay low VAF sequences from human genomic DNA in the condition of wild-type (WT)-specific sink. The binding affinity for SNV sequence was improved by at least 35 -fold than previous selective detection systems, such as molecular beacons, Yin-yang probes, and toehold probes. Nevertheless, they focused mainly on improving the hybridization specificity, and the molecular sensitivity was not so satisfying for practical application. Besides, the involvement of sink made the method complex. Therefore, it is still necessary and meaningful to develop a simple and robust method with better specificity and sensitivity for SNV detection.

At the same time, nucleic acid analogs, as diagnostic agents and research tools, have become increasingly significant in the field of biosensors due to their chemical stability, high selectivity, and 
affinity towards complementary DNA/RNA. The use of nucleic acid analogs, such as piptide nucleic acid and locked nucleic acid (LNA), has undergone a significant upsurge of interest [32-34]. Using LNA as substitution, the affinity of sequence-specific binding to complementary single stranded DNA or RNA dramatically increased, and the melting temperatures increased by as much as $10{ }^{\circ} \mathrm{C}$ with each LNA substitution $[35,36]$, which makes LNA an excellent tool for SNV analysis [37]. Additionally, LNA has several peculiar properties, including enhanced nuclease resistance, non-detectable toxicity in vivo, and commercial availability [38]. These merits of LNA make it a very flexible substitution in nucleic acid-based detection technologies.

Spurred on by all the above findings, herein, an enzyme-free and sensitive fluorescence biosensor based on both LNA-integrated X-shaped probe and TMSDR-assisted recycling amplification system was developed for SNV detection. The target sequence used in this study is $\beta$-thalassemia mutation gene, a site-specific mutation of adenine to guanine (c. $-78 \mathrm{~A}>\mathrm{G}$ ). As is well known, $\beta$-thalassemia is a common hereditary anemia worldwide including Southern China, which is a group of single-gene genetic disorders deriving from more than 200 point mutations in the $\beta$-hemoglobin gene on chromosome 11 [39]. The high gene frequency of $\beta$-thalassemia 
affects multiple organs and is associated with considerable morbidity and mortality $[40,41]$. Accordingly, it is vital and urgent to construct a new method for the genetic diagnosis of $\beta$-thalassemia. Satisfactorily, in our strategy, specificity was improved by substituting LNA for sink reported by Wang [31], and high sensitivity was also obtained by incorporating TMSDR-assisted recycling signal amplification. The detection limit was down to $6 \mathrm{fM}$ and capable of detecting concentrations of variant alleles as low as $0.01 \%$.

\section{Experimental Section}

\subsection{Reagents and apparatus}

All oligonucleotides were HPLC purified and synthesized by Sangong Biotech Co., Ltd (Shanghai, China). The sequences of oligonucleotides were listed in Table S1 (Supplementary Information) and their concentrations were quantified by OD260 based on their individual absorption coefficients. $5 \times$ Phosphate Buffered Saline (5xPBS) used in this study was diluted from $10 \times \mathrm{PBS}$ which purchased commercially from Sigma-Aldrich (USA). The serum sample was obtained from the Union Hospital of Fujian Medical University (Fujian, China). All other reagents were of analytical grade and all aqueous solutions were prepared with Milli-Q water $(18 \mathrm{M} \Omega \cdot \mathrm{cm})$. 
The fluorescence spectra were measured on a Cary Eclipse fluorescence spectrometer from Agilent Technologies (USA). The studied excitation wavelength was $494 \mathrm{~nm}$ with $5 \mathrm{~nm}$ excitation and emission slits.

\subsection{X-shaped DNA probe preparation and formation}

The preparation of $\mathrm{X}$-shaped DNA probes can be readily accomplished according to the reference [31]. In brief, mixing together the $\mathrm{Q}, \mathrm{F}, \mathrm{C}$ and $\mathrm{P}$ strands in 5:1:1.5:3 ratio, respectively, in a solution that was finally $1 \mu \mathrm{M}$ in the limiting reagent $(\mathrm{F})$ and $5 \times$ Phosphate Buffered Saline. The mixtures of above four strands were then thermally annealed using a personal PCR machine, went through a process of initial heating to $95{ }^{\circ} \mathrm{C}$ for 5 minutes, and cooling to $20{ }^{\circ} \mathrm{C}$ over the course of next 75 minutes. The obtained solution was preserved at $4{ }^{\circ} \mathrm{C}$ until use.

\subsection{Fluorescence analysis}

$\mathrm{X}$-shaped DNA probes were first incubated with different concentration SNV and $1.5 \mu \mathrm{M}$ of two auxiliary complementary strands at $40{ }^{\circ} \mathrm{C}$ for 60 minutes. Then, at room temperature, the solutions were removed into a spectrofluorometer cuvette with a transferpettor. The fluorescence spectrum was subsequently taken in the wavelength range $500 \mathrm{~nm}$ to $620 \mathrm{~nm}$ at the $494 \mathrm{~nm}$ excitation wavelength and the emission wavelength were obtained at $515 \mathrm{~nm}$. 
All the experiments were operated for at least 3 times, and the values were averaged from at least 3 independent experiments.

\subsection{The preparation of artificial serum sample}

Blood sample was collected in coagulant tube and centrifuged for $5 \mathrm{~min}$ at $3000 \mathrm{rpm}$ after $1 \mathrm{~h}$ standing. The supernatant liquid, i.e., serum was transferred and stored in the liquid nitrogen. Then, $1 \mathrm{~mL}$ of serum was taken and diluted to $10 \mathrm{~mL}$ with $5 \times \mathrm{PBS}$. The different concentrations of mutDNA were then added into the diluted serum to prepare artificial serum samples.

\section{Results and discussion}

\subsection{Principle of the proposed fluorescence biosensor}

The detailed principle of the assay strategy and DNA sensor architecture were illustrated in Figure 1. As is denoted by number, each strand can be subdivided into various regions, expressing as a number of successive nucleotides and acting as the functional units in hybridization, dissociation or branch migration; an asterisk region denotes a region complementary in sequence to the region without it (for example, Region $1^{*}$ is complementary to Region 1 ). The X-shaped probe comprises four components (see Figure 1): a universal quencher strand Q (containing two domains termed as Regions 1 and 2), a universal fluorophore strand F (containing two 
domains termed as Regions 3 and $2 *$ ), a specific complementary strand C (containing four domains termed as Regions $6,5^{*}, 4^{*}$, and $3^{*}$ ), and a specific protector strand $\mathrm{P}$ (containing three domains termed as Regions $1^{*}, 4$, and 5).

The hybridization between Region 2 and Region $2 *$ results in the quencher being in close proximity to the fluorophore, so that the $\mathrm{X}$-shaped probe is natively dark. Initiated by region 6 (known as a toehold, containing a LNA location to improve the affinity), the reaction between $\mathrm{X}$-shaped probe and its target goes through a branch migration process. The reaction is completed via the dissociation of regions 4 and 2, and thus the PQ complex or product $\mathrm{B}$ is liberated at the same time. Then, two auxiliary specific single-stranded DNA probes AP1 and AP2 (containing two LNA locations which would enhance the efficiency of strand-displacement reaction) are introduced to hybridize, respectively, with Strand F (Regions $2^{*}$ and 3 ) and $\mathrm{C}$ (Regions $3 *, 4^{*}$ and $5^{*}$ ), leading to the generation of $\mathrm{D}$ and $\mathrm{E}$, and the release of target. Subsequently, the released target further hybridizes with another Regions 6 and 5*, which induces the recycling of strand-displacement reaction between the X-shaped probe and target. After numerous cycles, theoretically, all the X-shaped probes are dissociated and F strands are all liberated, resulting in an obvious enhancement of the fluorescent signal. 
Consequently, an enzyme-free and sensitive fluorescence biosensor for SNV detection was achieved. Obviously, this protocol shows some advantages: (i) the architecture of $\mathrm{X}$-shaped probe can target different SNV sequence because its functionalized oligonucleotides could be decoupled from the target-specific regions and thereby would avoid the high costs of oligonucleotide-synthesis [42-44]; (ii) the use of LNA in X-shaped DNA probe improves the stability and specificity of the sensor, but avoiding the complex design process of sink; (iii) the introduction of TMSDR-assisted recycling amplification system improves the sensitivity of the sensor.

Figure 1 should be inserted in here

\subsection{Feasibility tests of discrimination for $S N V$}

The feasibility of our strategy for SNV discrimination was confirmed by comparing fluorescence spectra obtained from the different targets of random DNA, wtDNA, mutDNA and no mutDNA (blank). As can be seen in Figure 2, mutDNA causes intense fluorescence signal, but for the wtDNA even on the basis of only one-base difference, fluorescence signal is very weak. The fluorescence intensity for $1 \mathrm{pM}$ mutDNA is about 37 times as large as that for wtDNA at the same concentration. What's more, random DNA and blank only generate negligible responses. These results demonstrate that the TMSDR can be only achieved by using the 
probe exactly matched with mutDNA, which can further perform the recycling amplification in the reaction system, indicating an excellent recognition ability of this method for SNV detection. By substituting corresponding nucleic acid for LNA, recognition ability of the non-LNA X-shaped probe for random DNA, wtDNA and mutDNA was also studied at the same condition (See Figure S1). It was found that when there was an absence of LNA in X-shaped probe, random DNA and wtDNA caused much higher fluorescence signals comparing with the LNA-integrated probe. These results demonstrate that the LNA can improve the specificity of the sensor. In addition, the feasibility of this strategy was further confirmed by gel electrophoresis (See Figure S2).

Figure 2 should be inserted in here

\subsection{Optimizations of experimental parameters}

For the best sensing performance of the fluorescence sensor, important experimental parameters, such as temperature and incubation time, were investigated. The hybridization temperature of the detection system, which can affect both hybridization and cycle efficiency of DNA directly, was firstly investigated as a key factor. The fluorescence intensity increases firstly to the maximum value at around $40{ }^{\circ} \mathrm{C}$ and then decreases as the temperature rises (Figure S3). Therefore, $40{ }^{\circ} \mathrm{C}$ was selected for subsequent experiments. The 
fluorescence intensity can also be influenced by the incubation time. The time required for fluorescence intensity to reach the steady value is around or over 60 minutes, and thereby 60 minutes was chosen for further studies (Figure S4).

\subsection{Sensitivity of the fluorescence biosensor}

To acquire the quantitative values of the fluorescence sensor, mutDNA of varying concentrations were measured by using fluorescence spectra under the optimized experimental conditions. As can be seen from Figure 3, only a quite weak fluorescence signal is observed in the absence of mutDNA while obvious fluorescence signal is expressed when the concentration of mutDNA is $10 \mathrm{fM}$, suggesting the high signal-to-background of this method. The fluorescence intensities are linearly dependent on the mutDNA concentration in a wide range from $10 \mathrm{fM}$ to $1 \mathrm{pM}$. The correlation equation is: $\mathrm{F}=0.1163 \mathrm{C}+19.192$ with a correlation coefficient of 0.9950. The detection limit, based on $3 \sigma /$ slope, was calculated to be $6 \mathrm{fM}$ (showed in Supplementary Table S2). The relative standard deviation (RSD) was estimated to be $2.9 \%$ for five replicate determinations with the same set of reagents of $1000 \mathrm{fM}$ mutDNA. Thus, this proposed approach is much more sensitive than previous method (listed in Supplementary Table S3). One of the main important reasons for the high sensitivity of this approach is the 
introduction of TMSDR-assisted recycling amplification system, which takes place without enzymes at room temperature, and hence enhances the sensitivity of hybridization extremely. In addition, the fluorescence signals for different times have been also tested and the RSD is $5.7 \%$, which is acceptable for instrumental analysis.

Figure 3 should be inserted in here

\subsection{Specificity of the fluorescence biosensor}

High specificity is another important aspect to evaluate an analysis method. To interrogate the specificity of this method, some mixed DNA samples have been prepared, which contain wtDNA and mutDNA at various molar ratios of 100:1, 200:1, 500:1, 1000:1, 2000:1, 5000:1 and 10,000:1 with a total concentration of $0.1 \mathrm{nM}$. Here, the blank sample only contains $0.1 \mathrm{nM}$ wtDNA. These mixed DNA samples, serving as the target, can cause the proposed cycle reaction by react with $\mathrm{X}$-shaped probes that are complementary to the mutDNA. From Figure 4, it can be observed that the fluorescence signals are dynamically enhanced with the increase of the mutDNA concentration in mixed DNA samples. It is noticeable that even the mutDNA concentration is as low as $10 \mathrm{fM}$, positive control signals still could be discriminated from the blank. In other words, rare SNV detection was as low as $0.01 \%$ VAF by using our sensor. These experimental results demonstrate that our developed 
method possesses extremely high specificity and sensitivity for $\beta$-thalassemia SNV detection and may meet the requirement of the actual sample analysis.

Figure 4 should be inserted in here

\subsection{The analysis of artificial serum sample}

With respect to a complex sample analysis system, to demonstrate the practicability of the proposed method, a serial of artificial serum samples with three concentrations of mutDNA (50, $500,1000 \mathrm{fM}$ ) were determined by employing this proposed method. As expressed in Table 1, the recovery was between 93.6 to $110.0 \%$. On this point, the proposed method shows the potentiality for SNV detection in real complex biological matrices.

\section{Table 1 should be inserted in here}

\section{Conclusion}

In summary, an enzyme-free and sensitive fluorescence biosensor was developed for the direct detection of SNV. This novel approach integrates the advantages of X-shaped probe, LNA and TMSDR-assisted recycling amplification for target SNV recognition, resulting in the excellent specificity and high sensitivity. The proof-of-concept has illustrated the feasibility and potential applicability of this strategy for sensitive and selective detection of 
$\beta$-thalassemia mutant genes. Moreover, this proposed fluorescence biosensor can readily be extended to the SNV detection of other malignant tumors by altering corresponding probe sequences. Of course, the embedded equipment could be more robust, cost-efficient, and readily automated because of its homogeneous and fluorescence-based detection format.

\section{Acknowledgements}

The authors grateful acknowledge the financial support of the National Natural Science Foundation of China (21375017, 21105012), the Natural Science Foundation of Fujian Province, China (2015J01596), the Education Department of Fujian Province, China (JA13149), the Medical Elite Cultivation Program of Fujian, P.R.C (2014-ZQN-ZD-26), the National Science Foundation for

Distinguished Young Scholars of Fujian Province (2013J06003), Program for New Century Excellent Talents of Colleges and Universities in Fujian Province (JA13130). 


\section{References}

[1] E.N. Imyanitov, Gene polymorphisms, apoptotic capacity and cancer risk, Hum. Genet. 125 (2009) 239-246.

[2] M.D. Gobbi, V. Viprakasit, J.R. Hughes, C. Fisher, V.J. Buckle, H. Ayyub, R.J. Gibbons, D. Vernimmen, Y. Yoshinaga, P.D. Jong, J.F. Cheng, E.M. Rubin, W.G. Wood, D. Bowden, D.R. Higgs, A regulatory SNP causes a human genetic disease by creating a new transcriptional promoter, Science 312 (2006) 1215-1217.

[3] H. Schwarzenbach, D.S.B. Hoon, K. Pantel, Cell-free nucleic acids as biomarkers in cancer patients, Nat. Rev. Cancer 11 (2011) 426-437.

[4] Z. Altintas, I.E. Tothill, DNA-based biosensor platforms for the detection of TP53 mutation, Sens. Actuators B: Chem. 169 (2012) 188-194.

[5] B. Vogelstein, N. Papadopoulos, V.E. Velculescu, S. Zhou, L.A. Diaz, K.W. Kinzler, Cancer genome landscapes, Science 339 (2013) 1546-1558.

[6] A.C. Syvanen, Accessing genetic variation: genotyping single nucleotide polymorphisms, Nat. Rev. Genet. 2 (2001) 930-942.

[7] J.A. Prince, L. Feuk, W.M. Howell, M. Jobs, T. Emahazion, K. Blennow, A.J. Brookes, Robust and accurate single nucleotide polymorphism genotyping by dynamic allele-specific 
hybridization (DASH): design criteria and assay validation, Genome Res. 11 (2001) 152-162.

[8] J. Li, S. Schachermeyer, Y. Wang, Y. Yin, W. Zhong, Detection of microRNA by fluorescence amplification based on cation-exchange in nanocrystals, Anal. Chem. 81 (2009) 9723-9729.

[9] Y. Cheng, Q. Du, L. Wang, H. Jia, Z. Li, Fluorescently cationic conjugated polymer as an indicator of ligase chain reaction for sensitive and homogeneous detection of single nucleotide polymorphism, Anal. Chem. 84 (2012) 3739-3744.

[10] W. Shen, C.L. Lim, Z. Gao, A ferrofluid-based homogeneous assay for highly sensitive and selective detection of single-nucleotide polymorphisms, Chem. Commun. 49 (2013) 8114-8116.

[11] S. Shin, B.Y. Won, C. Jung, S.C. Shin, D.Y. Cho, S.S. Lee, H.G. Park, Electrochemical detection of DNA mutations on a PNA-modified electrode utilizing a single-stranded DNA specific endonuclease, Chem. Commun. 47 (2011) 6611-6613.

[12] H. Zhou, S.J. Xie, S.B. Zhang, G.L. Shen, R.Q. Yu, Z.S. Wu, Isothermal amplification system based on template-dependent extension, Chem. Commun. 49 (2013) 2448-2450.

[13] X. Duan, S. Wang, Z. Li, Conjugated polyelectrolyte-DNA 
complexes for multi-color and one-tube SNP genotyping assays, Chem. Commun. (2008) 1302-1304.

[14] J. Gaster, G. Rangam, A. Marx, Increased single nucleotide discrimination in arrayed primer elongation by 49C-modified primer probes, Chem. Commun. (2007) 1692-1694.

[15] F. Chen, E.A. Gaucher, N.A. Leal, D. Hutter, S.A. Havemann, S. Govindarajan, E.A. Ortlund, S.A. Benner, Reconstructed evolutionary adaptive paths give polymerases accepting reversible terminators for sequencing and SNP detection, PNAS 107 (2010) $1948-1953$.

[16] P. Cahill, M. Bakis, J. Hurley, V. Kamath, W. Nielsen, D. Weymouth, J. Dupuis, L. Doucette-Stamm, D.R. Smith, Exo-proofreading, a versatile SNP scoring technology, Genome Res. 13 (2003) 925-931.

[17] Y.J. Hua, Z.F. Li, A.M. Diamond, Enhanced discrimination of single nucleotide polymorphism in genotyping by phosphorothioate proofreading allele-specific amplification, Anal. Biochem. 369 (2007) 54-59.

[18] A.X. Zheng, J. Li, J.R. Wang, X.R. Song, G.N. Chen, H.H. Yang, Enzyme-free signal amplification in the DNAzyme sensor via target-catalyzed hairpin assembly, Chem. Commun. 48 (2012) $3112-3114$ 
[19] X. Zhang, J. Zhang, D. Wu, Z. Liu, S. Cai, M. Chen, Y. Zhao, C.

Li, H. Yang, J. Chen, Ultraselective electrochemiluminescence biosensor based on locked nucleic acid modified toehold-mediated strand displacement reaction and junction-probe, Analyst 139 (2014) 6109-6112.

[20] X. Wang, M. Zou, H. Huang, Y. Ren, L. Li, X. Yang, N. Li, Gold nanoparticle enhanced fluorescence anisotropy for the assay of single nucleotide polymorphisms (SNPs) based on toehold-mediated strand-displacement reaction, Biosens. Bioelectron. 41 (2013) 569-575.

[21] J.B. Long, Y.X. Liu, Q.F. Cao, Q.P. Guo, S.Y. Yan, X.X. Meng, Sensitive and enzyme-free detection for single nucleotide polymorphism using microbead-assisted toehold-mediated strand displacement reaction, Chin. Chem. Lett. 26 (2015) 1031-1035.

[22] D. Wang, W. Tang, X. Wu, X. Wang, G. Chen, Q. Chen, N. Li, F. Liu, Highly selective detection of single-nucleotide polymorphisms using a quartz crystal microbalance biosensor based on the toehold-mediated strand displacement reaction, Anal. Chem. 84 (2012) 7008-7014.

[23] C. Li, Y. Li, X. Xu, X. Wang, Y. Chen, X. Yang, F. Liu, N. Li, Fast and quantitative differentiation of single-base mismatched DNA by initial reaction rate of catalytic hairpin assembly, Biosens. 
Bioelectron. 60 (2014) 57-63.

[24] Y. Guo, J. Wu, H. Ju, Target-driven DNA association to initiate cyclic assembly of hairpins for biosensing and logic gate operation, Chem. Sci. 6 (2015) 4318-4323.

[25] Y.S. Jiang, S. Bhadra, B. Li, A.D. Ellington, Mismatches improve the performance of strand-displacement nucleic acid circuits, Angew. Chem. Int. Ed. 53 (2014) 1845-1848.

[26] C. Buranachai, S.A. McKinney, T. Ha, Single molecule nanometronome, Nano Lett. 6 (2006) 496-500.

[27] D.M. Kolpashchikov, A binary DNA probe for highly specific nucleic acid recognition, J. Am. Chem. Soc. 128 (2006) 10625-10628.

[28] Y.V. Gerasimova, D.M. Kolpashchikov, Detection of bacterial 16S rRNA using a molecular beacon-based $\mathrm{X}$ sensor, Biosens. Bioelectron. 41 (2013) 386-390.

[29] D.M. Kolpashchikov, Y.V. Gerasimova, M.S. Khan, DNA nanotechnology for nucleic acid analysis: DX motif-based sensor, Chembiochem. 12 (2011) 2564-2567.

[30] C.C. Lee, Y.C. Liao, Y.H. Lai, M.C. Chuang, Recognition of dual targets by a molecular beacon-based sensor: subtyping of influenza a virus, Anal. Chem. 87 (2015) 5410-5416.

[31] J.S. Wang, D.Y. Zhang, Simulation-guided DNA probe design 
for consistently ultraspecific hybridization, Nat. Chem. 7 (2015) $545-553$.

[32] K. Bondensgaard, M. Petersen, S.K. Singh, V.K. Rajwanshi, R. Kumar, J. Wengel, J.P. Jacobsen, Structural studies of LNA: RNA duplexes by NMR: conformations and implications for RNase $\mathrm{H}$ activity, Chem. Eur. J. 6 (2000) 2687-2695.

[33] L. Lin, J. Kang, S. Weng, J. Chen, A. Liu, X. Lin, Y. Chen, Locked nucleic acids biosensor for detection of BCR/ABL fusion gene using benzoate binuclear copper (II) complex as hybridization indicator, Sens. Actuators B: Chem. 155(2011) 1-7.

[34] P. Kumar, M.E. Østergaard, B. Baral, B.A. Anderson, D.C. Guenther, M. Kaura, D.J. Raible, P.K. Sharma, P.J. Hrdlicka, Synthesis and biophysical properties of C5-functionalized LNA (locked nucleic acid), J. Org. Chem. 79 (2014) 5047-5061.

[35] D.A. Braasch, D.R. Corey, Locked nucleic acid (LNA): fine-tuning the recognition of DNA and RNA, Chem. Biol. 8 (2001) 1-7.

[36] G. Suresh, U.D. Priyakumar, Structures, dynamics, and stabilities of fully modified locked nucleic acid $(\beta$-d-LNA and $\alpha$-l-LNA) duplexes in comparison to pure DNA and RNA duplexes, J. Phys. Chem. B 117 (2013) 5556-5564.

[37] Z.F. Gao, Y. Ling, L. Lu, N.Y. Chen, H.Q. Luo, N.B. Li, 
Detection of single-nucleotide polymorphisms using an ON-OFF switching of regenerated biosensor based on a locked nucleic acid-integrated and toehold-mediated strand displacement reaction, Anal. Chem. 86 (2014) 2543-2548.

[38] J. Chen, J. Zhang, K. Wang, X. Lin, L. Huang, G. Chen, Electrochemical biosensor for detection of $\mathrm{BCR} / \mathrm{ABL}$ fusion gene using locked nucleic acids on 4-aminobenzene, Anal. Chem. 80 (2008) 8028-8034.

[39] P. Yi, F. Yu, S. Huang, C. Zhong, Q. Li, Y. Yang, W. Zhang, C. Xiao, X. Xu, Identification of a novel frameshift mutation at codon $53(-\mathrm{T})$ in the $\beta$-globin gene causing dominantly inherited $\beta$-thalassemia in a Chinese Miao family, Blood Cell. Mol. Dis. 41 (2008) 56-59.

[40] D. Rund, E. Rachmilewitz, $\beta$-thalassemia, N. Engl. J. Med. 353 (2005) 1135-1146.

[41] M. Lin, J.W. Jiao, X.H. Zhan, X.F. Zhan, M.C. Pan, J.L. Wang, C.F. Wang, T.Y. Zhong, Q. Zhang, X. Yu, J.R. Wu, H.T. Yang, F. Lin, X. Tong, H. Yang, G.C. Zha, Q. Wang, L. Zheng, Y.F. Wen, L.Y. Yang, High resolution melting analysis: a rapid screening and typing tool for common $\beta$-thalassemia mutation in Chinese population, PLoS One 9 (2014) e102243-e102243.

[42] Y.V. Gerasimova, A. Hayson, J. Ballantyne, D.M. 
Kolpashchikov, A single molecular beacon probe is sufficient for the analysis of multiple nucleic acid sequences, Chembiochem. 11 (2010) 1762-1768.

[43] K. Knez, D. Spasic, K.P. Janssen, J. Lammertyn, Emerging technologies for hybridization based single nucleotide polymorphism detection, Analyst 139 (2014) 353-370.

[44] H. Ravan, Translating nucleic-acid hybridization into universal DNA-reporter sequences, Trends Anal. Chem. 65 (2015) 97-106. 


\section{Figure Captions}

Figure 1. Schematic illustration of the fluorescence biosensor and structure of the universal LNA-integrated X-shaped DNA probe.

Figure 2. Feasibility investigation: fluorescence spectra of no mutDNA (background, curve a), random DNA (curve b), wtDNA (curve c) and mutDNA (curve d). Insert: Bars represent the fluorescence intensity of no mutDNA, random DNA, wtDNA and mutDNA as targets, respectively. The concentration of each target analyte in the sample is $1 \mathrm{pM}$. Error bars were the standard deviations of triplicate measurements.

Figure 3. Fluorescence spectra of the sensor with different mutDNA concentrations $(0,6,10,100,300,400,500,600,800$, and $1000 \mathrm{fM}$, from bottom to top) at $494 \mathrm{~nm}$ excitation wavelength. Insert: the linear relationship between fluorescence intensity and mutDNA concentration. Error bars were the standard deviations of triplicate measurements.

Figure 4. Fluorescence intensities of mixed DNA samples at varying ratios of wtDNA to mutDNA. Error bars were the standard deviations of triplicate measurements. 


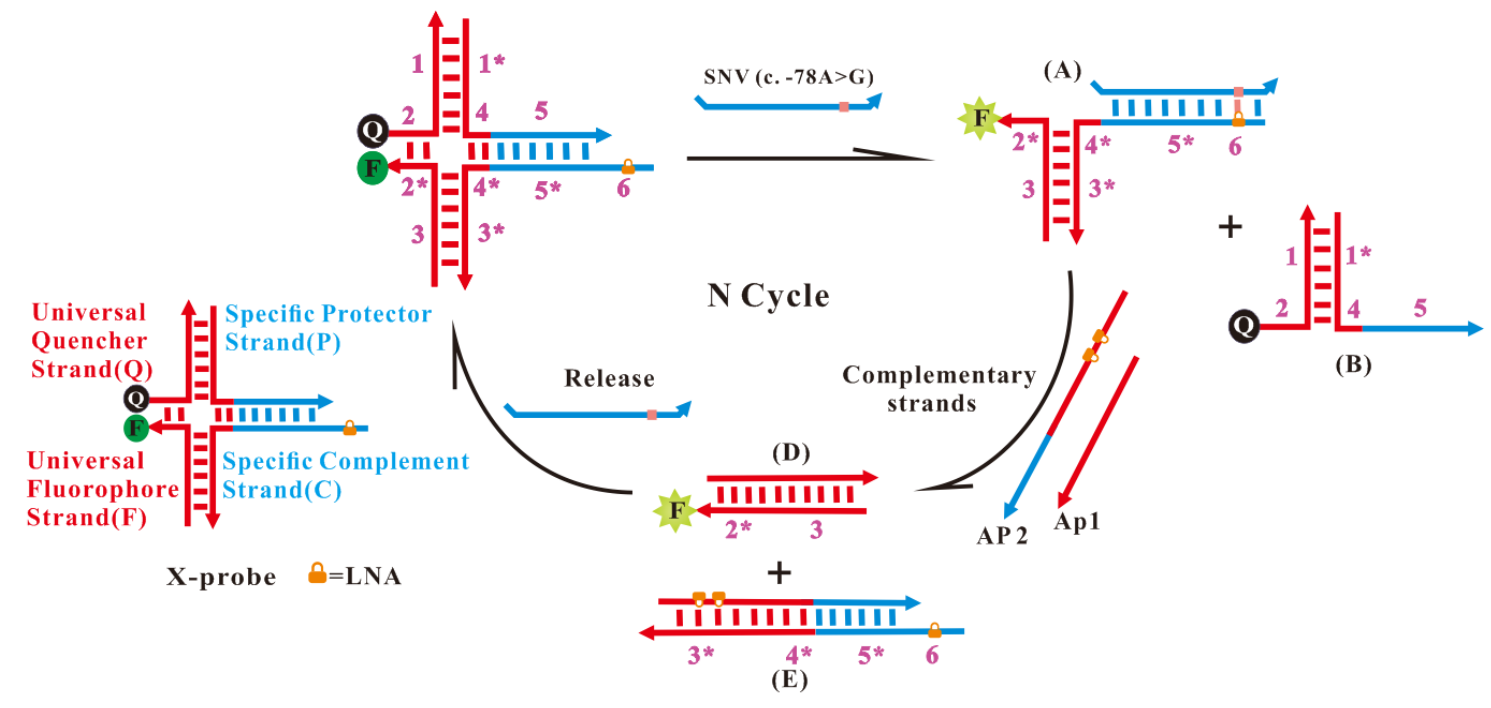

Figure 1.

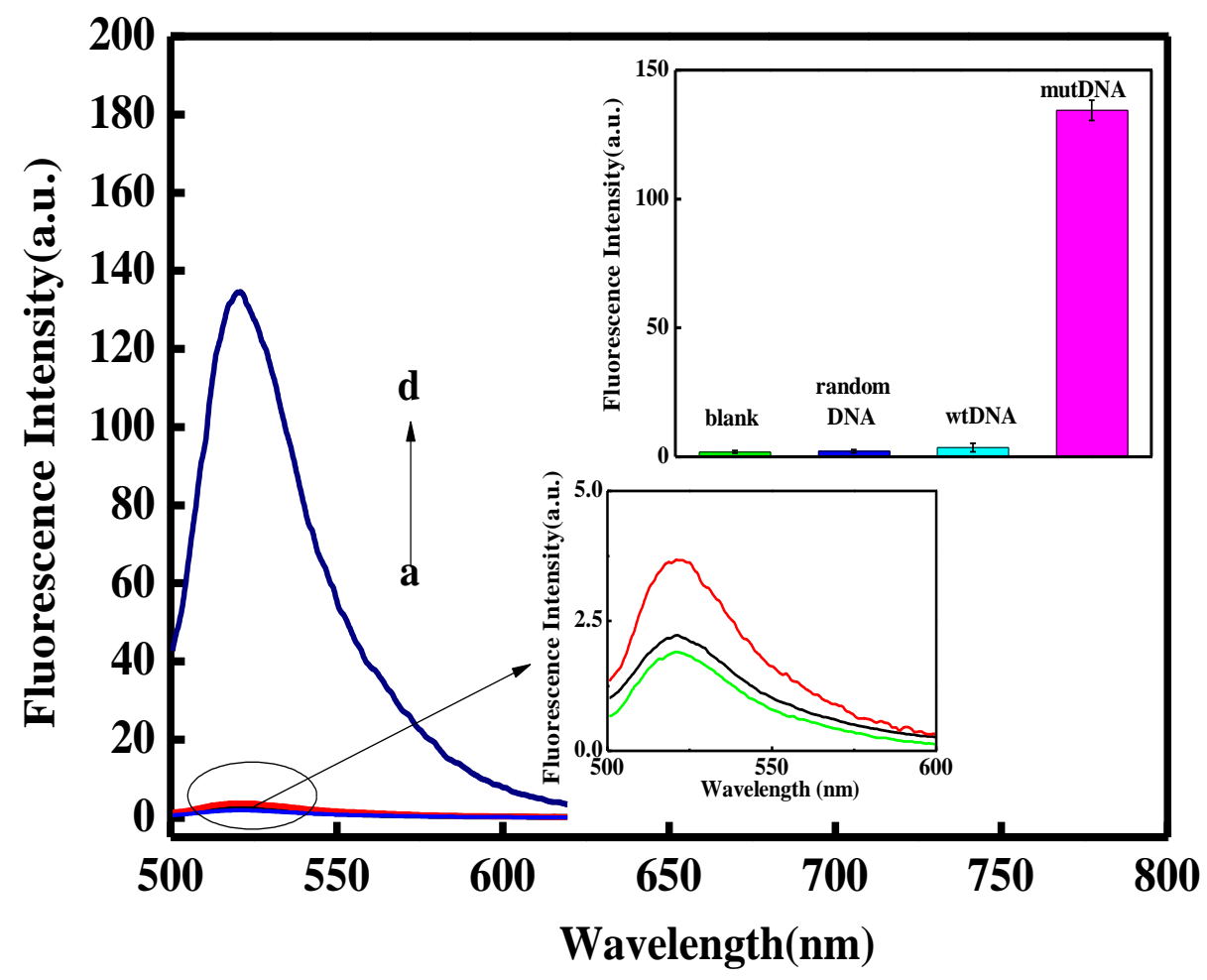

Figure 2. 


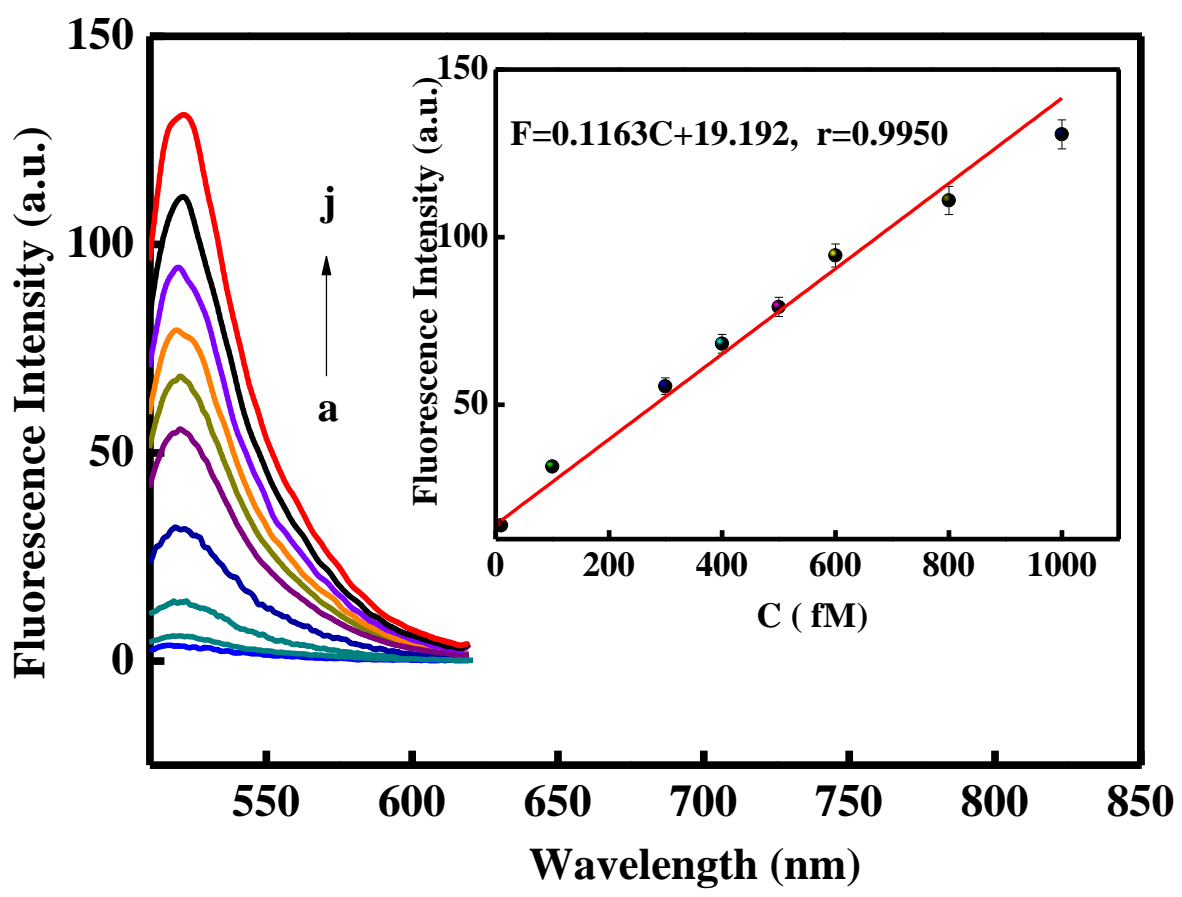

Figure 3.

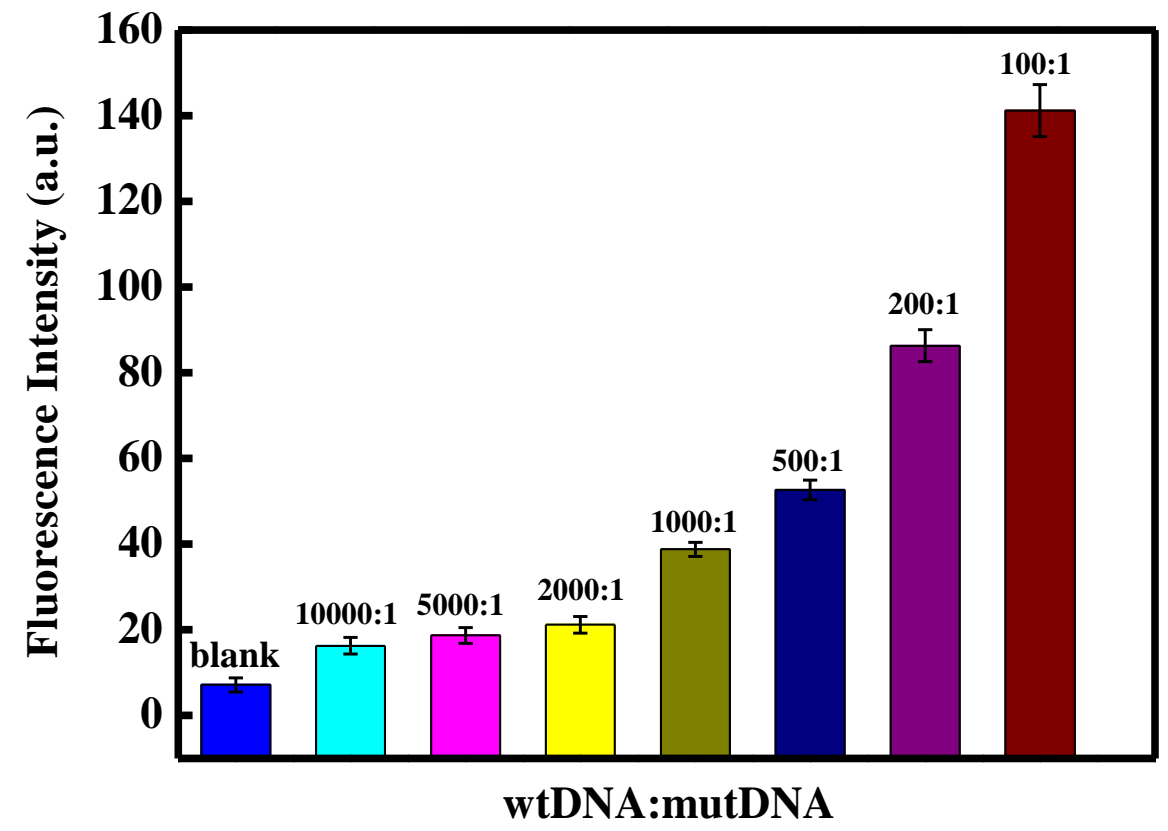

Figure 4. 


\section{Table Captions}

Table 1 Analysis results of the artificial synthetic specimens in serum $(\mathbf{n}=5)$

Table 1

\begin{tabular}{ccccc}
\hline & Sample no. & Added (fM) & Detected (fM) & Recovery (\%) \\
\hline \multirow{3}{*}{ mutDNA } & 1 & 50 & 55 & 110.0 \\
& 2 & 500 & 468 & 93.6 \\
& 3 & 1000 & 954 & 95.4 \\
\hline
\end{tabular}


Biographies

Fang Wu completed her doctorate at the Fuzhou University, Fujian province, China in 2012. She currently is an associate professor in Fujian Medical University, Fujian, China. Her main research interests are DNA biosensors development and sample pretreatment. Mei Chen obtained her bachelor degree in pharmacy from Fujian Medical University, Fujian province, China, in July 2013. She currently is a master student majoring in pharmaceutical analysis, Fujian Medical University, Fujian, China. Her research works are development and applications of DNA biosensor.

Jianming Lan completed his doctorate at the Fuzhou University, Fujian province, China in 2015. He currently is a lecturer in Fujian Medical University, Fujian, China. His main research interests are nanomaterials synthesis and applications in DNA biosensors development and biomedical diagnosis.

Yaokun Xia received his bachelor degree in pharmacy from Fujian Medical University, Fujian province, China in July 2013. In September 2014, he was enrolled as a master student in pharmaceutical analysis, Fujian Medical University, Fujian, China. His research work focuses on development and applications of DNA biosensors in various fields.

Mengmeng Liu obtained her bachelor degree in pharmaceutical 
engineering from Shandong University of Traditional Chinese Medicine, Shandong province, China, in July 2015. In September 2015, she was enrolled as a master student in pharmaceutical analysis, Fujian Medical University, Fujian, China. Her research interests are development and applications of DNA biosensors.

Wenhui He received her bachelor degree in traditional Chinese medicine from Hubei University of Chinese Medicine, Hubei province, China, in July 2015. She currently is a master student majoring in pharmaceutical analysis, Fujian Medical University, Fujian, China. Her research interests are development and applications of DNA biosensors.

Chunyan Li completed her doctorate at the Fuzhou University, Fujian province, China in 2012. She currently is a professor in Fujian Medical University, Fujian, China. Her main research interests are functional nanomaterials synthesis and applications in biological medicine and active pharmaceutical ingredients analysis. She has published more than 20 peer-reviewed papers in international journals.

Xiaosong Chen completed his doctorate at the Fujian Medical University, Fujian province, China in 2015. He currently is an associate professor in Fujian Medical University, Fujian, China. His main research interests are proteins, nucleic acids, signaling 
pathways and biological tracers. He has published more than 10 peer-reviewed papers in international journals. He has contributed to the biological part of this study.

Jinghua Chen completed his doctorate at the Fuzhou University, Fujian province, China in 2010. He currently is a professor in Fujian Medical University, Fujian, China. His main research interests are nanomaterials synthesis and applications in food safety analysis, DNA biosensors development, and biomedical diagnosis. He has published more than 100 peer-reviewed papers in international journals. 